\title{
Intervención Neuropsicológica Educativa en Trastornos del Lenguaje oral y escrito
}

\author{
Cristina de la Peña Álvarez. Universidad Francisco de Vitoria \\ Recepción: 10 de Junio de 2017 | Aceptado: 16 de Junio de 2017 \\ Correspondencia: Cristina de la Peña Álvarez | Correo-e: cristina.delapena@ufv.es
}

\section{(iD) $0000-0003-1176-4981$}

Citar: De la Peña, C. (2017).Intervención Neuropsicológica Educativa en Trastornos del Lenguaje oral y escrito. ReiDoCrea, 6(2), 48-55.

\begin{abstract}
Resumen: Los trastornos del lenguaje oral y escrito son las alteraciones más frecuentes en la realidad educativa y se necesitan propuestas de intervención que ayuden a mejorar el rendimiento de este alumnado. La implicación del proceso lingüístico en el desarrollo del resto de procesos cognitivos superiores $y$ en las habilidades académicas hace que sea un tema fundamental para los distintos profesionales de la psicología y educación. En este trabajo se hace una propuesta de intervención neuropsicológica educativa para los trastornos del lenguaje oral y para los trastornos del lenguaje escrito, incluyendo herramientas tecnológicas y programa basados en evidencia científica. Consecuentemente, estos datos tienen interesantes repercusiones para la práctica educativa diaria dirigida al tratamiento del alumnado con alteraciones en el lenguaje oral y escrito.
\end{abstract}

Palabras clave: Neuropsicología | Trastornos del lenguaje

\section{Educational Neuropsychological Intervention in oral and written language disorders}

Abstract: Oral and written language disorders are the most frequent alterations in the educational reality and intervention proposals are needed that help to improve the performance of this students. The implication of the linguistic process in the development of the rest of higher cognitive processes and in the academic abilities makes it a fundamental subject for the different professionals of the psychology and education. In the present work a proposal of educational neuropsychological intervention for the oral language disorders and for the written language disorders is realized, including technological tools and program based on scientific evidence. Consequently, these data have interesting repercussions for the daily educational practice aimed at the treatment of students with alterations in oral and written language.

Keywords: Neuropsychology | Language disorders

\section{Introducción}

El proceso neurolingüístico es resultado de la interacción de diversidad de mecanismos cerebrales que permiten el desarrollo y consolidación del lenguaje oral y escrito, en la modalidad de expresión y comprensión. Cualquier disfunción en alguno de estos mecanismos neuroanatómicos produce alteraciones en la recepción o producción del lenguaje oral y/o escrito. Para Pradas \& De la Peña (2016) las estructuras implicadas en la recepción del lenguaje, entre otras, son: el sistema auditivo, receptores sensitivos, nervios craneales y sistema nervioso central (estructuras corticales subcorticales); y, las estructuras neuroanatómicas que intervienen en la producción lingüística son, entre otras: el aparato fonoarticulatorio, nervios craneales y sistema nervioso central (a nivel cortical y subcortical).

Los Trastornos del Lenguaje Oral y Escrito están clasificados según el DSM-V (APA, 2013) dentro de la categoría Trastornos del Neurodesarrollo. Concretamente, las alteraciones del lenguaje oral aparecen con la denominación de Trastornos de la Comunicación y los problemas del lenguaje escrito como Trastornos Específicos del Aprendizaje con manifestación en el proceso lector o Dislexia y en la escritura o Disgrafía. 
Para los profesionales de la educación realizar cuanto antes la intervención neuropsicológica en este tipo de problemáticas es fundamental debido a la influencia del lenguaje en el resto de procesos cognitivos superiores $y$, por ende, en el rendimiento académico del alumnado.

La intervención neuropsicológica descansa sobre la plasticidad cerebral y es fundamental que psicólogos y profesores conozcan la capacidad de modificar el aprendizaje, para diseñar estrategias que optimicen el proceso de enseñanza/aprendizaje. Concretamente, la intervención neuropsicológica educativa en los trastornos del lenguaje beneficia a todos los agentes educativos, tanto a los escolares para mejorar sus problemas, como a los profesores y psicólogos para optimizar sus respuestas educativas, como a los padres y a la propia administración educativa.

La realidad diaria en las aulas evidencia la necesidad de realizar distintas intervenciones neuropsicológicas debido a la diversidad de problemas lingüísticos presentes en el aula. Por esta razón, en primer lugar, se describe la intervención neuropsicológica educativa para los Trastornos del Lenguaje Oral y, en segundo lugar, la intervención neuropsicológica educativa para los Trastornos del Lenguaje Escrito.

\section{Intervención Neuropsicológica Educativa en Trastornos del Lenguaje Oral}

La heterogeneidad de los trastornos del Lenguaje Oral (Baldo \& Dronkers, 2006; Cappa, 2015; De Thorne, Hengst \& Hamilton, 2016; Moreno-Flagge, 2013; PeñaCasanova, 2001; Puyuelo \& Salavera, 2011), hace que se necesiten distintos programas de intervención dirigidos a la rehabilitación neuropsicológica y cognitiva de cada uno de ellos; además, las dificultades lingüísticas pueden ser, en ocasiones, secundarias a otros trastornos cognitivos y/o sensoriales. Las alteraciones del lenguaje oral pueden afectar a la recepción lingüística y/o a la producción lingüística, observándose como más frecuentes en el aula: dislalia, retraso simple del lenguaje, disfasia, disfemia y disartria. De la Peña \& Pradas (2016) en la figura 1 recogen una síntesis de diferentes problemas del lenguaje oral y el ámbito afectado.

\begin{tabular}{ll}
\hline \multicolumn{1}{c}{ Dificultad Linguística } & \multicolumn{1}{c}{ Ámbito afectado } \\
\hline Retraso Simple del Lenguaje & Retraso cronológico de todos los procesos del lenguaje \\
\hline Disfasia o Trastorno Específico del Lenguaje (TEL) & Alteración cualitativa en la expresión y/o comprensión \\
\hline Afasia & Todos los procesos del lenguaje \\
Wernicke: & $\begin{array}{l}\text { Comprensión } \\
\text { Expresión }\end{array}$ \\
Broca: & Articulación a nivel funcional \\
\hline Dislalia & Articulación por malformación anatómica \\
\hline Disglosia & Articulación por alteración del sistema nervioso central \\
\hline Disartria & Fluidez y ritmo del habla \\
\hline Tartamudez & Voz \\
\hline Disfonías & Prosodia \\
\hline Trastorno de prosodia &
\end{tabular}

Figura 1. Síntesis Alteraciones del Lenguaje infantil

Fuente: De la Peña \& Pradas (2016, p.167) 
Los trastornos lingüísticos conllevan consecuencias socioemocionales para el alumnado, por lo que se necesita una rápida intervención. En líneas generales, en la tabla 1 se propone un resumen de los procesos neuropsicológicos afectados en los distintos trastornos del Lenguaje Oral y ejemplos de ejercicios para trabajarlos en el aula.

\section{Tabla 1}

Resumen de procesos neuropsicológicos y ejercicios

\begin{tabular}{|c|c|}
\hline $\begin{array}{l}\text { Procesos } \\
\text { Neuropsicológicos }\end{array}$ & Tipos de Ejercicios \\
\hline Funcionalidad Auditiva & $\begin{array}{l}\text { Localizar un sonido, discriminar entre distintas secuencias de } \\
\text { ritmo }\end{array}$ \\
\hline Motricidad & Juego del twister y ejercicios de lateralidad \\
\hline Respiración & Soplar por una pajita, inflar un globo \\
\hline Orofaciales & Ejercicios de succión y masticación \\
\hline $\begin{array}{l}\text { Praxias linguales, labiales y } \\
\text { madnibulares }\end{array}$ & Poner la lengua, labios y mandíbula en distintas posiciones \\
\hline Lenguaje: & \\
\hline Conciencia fonológica & $\begin{array}{l}\text { Reconocer si dos palabras comienzan por el mismo } \\
\text { fonema }\end{array}$ \\
\hline $\begin{array}{l}\text { Proceso Léxico/ } \\
\text { Semántico }\end{array}$ & - $\quad$ Ante objetos decir su significado \\
\hline Procesos Sintáctico & Transformar frases \\
\hline Proceso Pragmático & Adaptar el lenguaje a cada situación dada \\
\hline Comprensión Oral & Realizar acciones según las indicaciones dadas \\
\hline Expresión Oral & Narrar acontecimientos, contar un cuento \\
\hline
\end{tabular}

La tecnología educativa facilita diversas posibilidades para realizar de forma lúdica y divertida la rehabilitación de los distintos trastornos lingüísticos. A continuación, se refieren algunos de los programas y/o aplicaciones tecnológicas más frecuentemente utilizadas en lenguaje oral:

- Programa Phonetics: este programa permite ver la articulación de los fonemas dentro de palabras o de forma aislada.

- Programa Visi-Pitch: es un analizador del tono que permite trabajar la entonación.

- Conversations Framework: mediante conversaciones entrena vocabulario, sintaxis, pragmática y organización del lenguaje.

- $\quad$ Talkin on the go: este programa permite mejorar la discriminación auditiva y la producción y expresión lingüística.

Intensive phonological awaraeness Program: trabaja la conciencia fonológica.

- Programa P.E.L.O.: este programa mediante ejercicios entrena articulación, conciencia fonológica, praxias, respiración, discriminación auditiva y ámbitos léxico y sintáctico.

- $\quad$ ArtikPix: trabaja la articulación.

- Language Therapy Lite- Toolkit for Aphasia: permite trabajar la Afasia en niños. 
- Programa Hamlet: este programa trabaja la conciencia fonológica con ejercicios de segmentación fonemática y silábica. logopedia.

Programa LAO: permite la creación de propios ejercicios relacionados con la

- $\quad$ Felt Board: trabaja el lenguaje expresivo y la comprensión oral.

Proloquo2Go: entrena el lenguaje receptivo.

- Programas Exler: mediante campos semánticos trabaja el componente semántico del lenguaje.

Programa E.A.R.: este programa entrena la conciencia fonológica y la articulación de distintos fonemas.

Speech Trainer 3D: este programa trabaja la articulación de vocales y consonantes.

- Go Togethers: entrena el proceso semántico mediante ejercicios de categorías semánticas.

- $\quad$ Programa Speech Viewer: es un visualizador fonético que permite trabajar la prosodia y conciencia fonológica.

- Expresive Builder: este programa trabaja el lenguaje expresivo y comprensivo.

- See.Touch.Learn: entrena el lenguaje oral receptivo.

WoodLadder: juego de palabras que consiste en cambiar y formar nuevas palabras a partir de las dadas.

Bob's Books: aplicación de juegos interactivos para trabajar la fonética, pronunciando palabras, deletreando y relacionando sonidos con palabras.

En síntesis, la intervención en Trastornos del Lenguaje Oral depende del tipo de problema lingüístico concreto y determinado, que condiciona los procesos afectados que se han de intervenir desde el punto de vista neuropsicológico, cognitivo y contando con la colaboración y coordinación de la familia y la escuela. Todo ello, utilizando la diversidad de programas y herramientas tecnológicas que permitan solventar las dificultades de los escolares y potenciar su formación integral, personal y educativa.

\section{Intervención Neuropsicológica Educativa en Trastornos del Lenguaje Escrito}

Dentro de los Trastornos del Lenguaje Escrito, este apartado se centra en las dificultades en el proceso lector o también conocido como Dislexia cuyas implicaciones se producen en el ámbito de la lectura y escritura; es la dificultad de aprendizaje más frecuente en las aulas y constituye una habilidad instrumental básica para el aprendizaje, de ahí la relevancia que la comunidad educativa confiere al tratamiento de este problema.

La aplicación de las técnicas de neuroimagen al estudio de la Dislexia, proporciona un mayor conocimiento y comprensión de esta dificultad de aprendizaje a los profesionales de la educación, cuya finalidad es optimizar la intervención neuropsicológica en el aula.

La literatura científica (Meiler et al., 2008; Shaywitz et al., 2004) evidencia que la intervención en Dislexia produce un aumento de activación en las regiones corticales que intervienen en la lectura. Esta intervención en el aula puede realizarse desde el tratamiento de los distintos ámbitos neuropsicológicos afectados, desde la aplicación de diferentes programas de rehabilitación con eficacia probada y desde la tecnología educativa aplicada a la dislexia. 
En relación a los procesos neuropsicológicos implicados en dislexia, en la tabla 2 se propone una síntesis - resumen de los procesos afectados y ejemplos de ejercicios para trabajarlos.

\section{Tabla 2}

Resumen de procesos neuropsicológicos y ejercicios

\begin{tabular}{|c|c|}
\hline $\begin{array}{l}\text { Procesos } \\
\text { Neuropsicológicos }\end{array}$ & Tipos de Ejercicios \\
\hline Funcionalidad Auditiva & $\begin{array}{l}\text { Discriminar sonidos o palabras diciendo si son iguales o } \\
\text { diferentes }\end{array}$ \\
\hline Funcionalidad Visual & Identificar una sílaba o palabra en un texto \\
\hline $\begin{array}{l}\text { Motricidad y desarrollo } \\
\text { vestibular }\end{array}$ & Conducir un balón entre obstáculos sin tocarlos \\
\hline Lateralidad & Reconocimiento derecha e izquierda en el papel \\
\hline Habilidades visoespaciales & Juego del Tangram \\
\hline Atención & Tareas de cancelación visual o auditiva \\
\hline Memoria & Reproducir un ritmo previamente escuchado \\
\hline Función Ejecutiva & Clasificar cartas según una instrucción dada \\
\hline $\begin{array}{ll}\text { Lenguaje: } \\
\text { - } & \text { Conciencia fonológica } \\
- & \text { Proceso Léxico } \\
- & \text { Proceso Semántico } \\
- & \text { Procesos Sintáctico } \\
- & \text { Proceso Pragmático } \\
- & \text { Comprensión Lectora } \\
- & \text { Comprensión Oral } \\
- & \text { Expresión Oral } \\
- & \text { Habilidades } \\
\text { metalingüísticas }\end{array}$ & $\begin{array}{ll}- & \text { Reconocer si dos palabras riman: sol y col } \\
- & \text { Identifica si una cadena de letras es o no una } \\
\text { palabra } & \\
- & \text { Decir el significado de ciertos objetos } \\
- & \text { Decidir si las frases son gramaticalmente correctas } \\
- & \text { Relacionar cada mensaje con su contexto concreto } \\
- & \text { Hacer frases de cada párrafo de un texto } \\
- & \text { Elegir una frase después de oírla } \\
- & \text { Narrar una historia a partir de viñetas } \\
- & \text { Estimar número de fonemas en canciones }\end{array}$ \\
\hline
\end{tabular}

En cuanto a los programas de rehabilitación para Dislexia, De la Peña (2016) recoge una síntesis de programas de intervención para Dislexia con los ámbitos de entrenamiento y los resultados obtenidos en los últimos diez años que se pueden observar en la figura 2. 


\begin{tabular}{|c|c|c|c|}
\hline Estudio & Sujetos & Ambito de intervención & Resultados \\
\hline $\begin{array}{l}\text { Kairaluoma, Ahonen, } \\
\text { Aro y Holopainen } \\
\text { (2007) } \\
\text { (finlandés) }\end{array}$ & $\begin{array}{l}2 \text { niños de } 7 \text { años con } \\
\text { dislexia }\end{array}$ & $\begin{array}{l}\text { Intervención en fluidez de lectura de } \\
\text { silabas, palabras y texto }\end{array}$ & $\begin{array}{l}\text { Mejora de la fluidez lectora a nivel } \\
\text { de silaba en los dos nínos. En uno } \\
\text { además mejoró en palabras y texto } \\
\text { y en el otro mejoró la precisión } \\
\text { lectora }\end{array}$ \\
\hline $\begin{array}{l}\text { Tressoldi, Vio y } \\
\text { lozzino (2007) } \\
\text { (italiano) }\end{array}$ & $\begin{array}{l}63 \text { alumnos, } 2^{\circ}-8^{\circ} \\
\text { grado, grupo control y } \\
\text { grupo experimental }\end{array}$ & $\begin{array}{l}\text { Intervención en fluidez lectora durante } 3 \\
\text { meses y con software. Reconocimiento de } \\
\text { sílabas dentro de palabras }\end{array}$ & $\begin{array}{l}\text { Mejora de la fluidez lectora en } \\
\text { ortografía regular }\end{array}$ \\
\hline $\begin{array}{l}\text { Soriano (2007) } \\
\text { (español) }\end{array}$ & $\begin{array}{l}\text { Grupo control ( } 15 \\
\text { niños) y grupo } \\
\text { experimental ( } 12 \text { niños } \\
\text { disléxicos) }\end{array}$ & $\begin{array}{l}\text { Intervención en fluidez lectora y } \\
\text { procesamiento fonológico con apoyo visual } \\
\text { de letras durante } 40 \text { sesiones de } 35 \\
\text { minutos (3sesiones/semana) }\end{array}$ & $\begin{array}{l}\text { Mejora de lectura de palabras y } \\
\text { pseudopalabras, aumento de } \\
\text { exactitud y velocidad lectora }\end{array}$ \\
\hline $\begin{array}{l}\text { Jiménez y Rojas } \\
\text { (2008) } \\
\text { (español) }\end{array}$ & $\begin{array}{l}62 \text { alumnos, } 9-12 \\
\text { años. Grupo control y } \\
\text { experimental }\end{array}$ & $\begin{array}{l}\text { Entrenamiento en conciencia fonológica y } \\
\text { reconocimiento de palabras a través del } \\
\text { videojuego Tradislexia }\end{array}$ & $\begin{array}{l}\text { Mejora de la conciencia fonológica } \\
\text { (síntesis y segmentación) y lectura } \\
\text { de pseudopalabras }\end{array}$ \\
\hline $\begin{array}{l}\text { Vadasy y Sanders } \\
\text { (2009) } \\
\text { (inglés) }\end{array}$ & $\begin{array}{l}202 \text { alumnos de } 2^{\circ} \text { y } \\
3^{\circ} \text { grado, grupo control } \\
\text { y grupo experimental }\end{array}$ & $\begin{array}{l}\text { Intervención en lectura repetida, durante } \\
15 \text { semanas en tutorias }\end{array}$ & $\begin{array}{l}\text { Mejora en fluidez, comprensión y } \\
\text { conocimiento de sonidos y letras }\end{array}$ \\
\hline $\begin{array}{l}\text { Suárez (2009) } \\
\text { (español) }\end{array}$ & 8 disléxicos, $7-12$ años & $\begin{array}{l}\text { Intervención en conciencia fonológica y } \\
\text { aprendizaje alfabético }\end{array}$ & $\begin{array}{l}\text { Mejora en exactitud y velocidad } \\
\text { lectora }\end{array}$ \\
\hline $\begin{array}{l}\text { Serrano y Defior } \\
\text { (2012) } \\
\text { (español) }\end{array}$ & $8-16$ años, 3 grupos & $\begin{array}{l}\text { Intervención en lectura repetida y } \\
\text { acelerada. } 24 \text { sesiones de } 1 \text { hora } \\
\text { (4sesiones/semana). } 4 \text { fases con } \\
\text { actividades de lectura de sílabas, palabras, } \\
\text { texto y letras y sonidos }\end{array}$ & $\begin{array}{l}\text { Mejora en velocidad y exactitud } \\
\text { lectora (palabras, pseudopalabras y } \\
\text { texto), en comprensión y } \\
\text { habilidades relacionadas con la } \\
\text { lectura }\end{array}$ \\
\hline $\begin{array}{l}\text { Matsuzawa y } \\
\text { Capellini (2012) } \\
\text { (portugués) }\end{array}$ & $\begin{array}{l}60 \text { alumnos, } 6-7 \text { años, } \\
\text { grupo control y } \\
\text { experimental }\end{array}$ & $\begin{array}{l}\text { Intervención en conciencia fonológica y } \\
\text { correspondencia grafema-fonema. } 18 \\
\text { sesiones de } 50 \text { minutos en clase }\end{array}$ & $\begin{array}{l}\text { Mejora en conciencia fonológica, } \\
\text { en habilidades cogntivas como } \\
\text { atención y memoria y en la } \\
\text { conversión grafema-fonema }\end{array}$ \\
\hline $\begin{array}{l}\text { Outon y Abal } \\
\text { (2013)(español) }\end{array}$ & $\begin{array}{l}1 \text { alumnos disléxico, } 7 \\
\text { años }\end{array}$ & $\begin{array}{l}\text { Intervencion multisensorial. } 30 \text { semanas y } \\
3 \text { sesiones/semana }\end{array}$ & $\begin{array}{l}\text { Mejora en precisión y fluidez } \\
\text { lectoras y en ortografia }\end{array}$ \\
\hline $\begin{array}{l}\text { Thomson, Leong y } \\
\text { Goswami (2013) } \\
\text { (inglés) }\end{array}$ & $\begin{array}{l}33 \text { disléxicos, } 9 \text { años, } \\
3 \text { grupos: uno control y } \\
\text { dos experimentales }\end{array}$ & $\begin{array}{l}\text { Intervención en ritmo a un grupo e } \\
\text { intervencion en discriminación fonética a } \\
\text { otro grupo }\end{array}$ & $\begin{array}{l}\text { Mejora en habilidades fonológicas } \\
\text { y prosódicas en los grupos } \\
\text { experimentales }\end{array}$ \\
\hline $\begin{array}{l}\text { Calvo, Calvo, Bueno, } \\
\text { Ruiz, Ballester y } \\
\text { Albacete (2014) } \\
\text { (español) }\end{array}$ & $\begin{array}{l}24 \text { disléxicos, 8-12 } \\
\text { años }\end{array}$ & $\begin{array}{l}\text { Intervención en fluidez lectora mediante } \\
\text { lectura ortográfica, comprensiva y } \\
\text { prosódica. } 20 \text { sesiones de } 25 \text { minutos en } \\
\text { clase y } 45 \text { sesiones de } 30 \text { minutos en casa }\end{array}$ & $\begin{array}{l}\text { Mejora de exactitud lectora, } \\
\text { velocidad y eficiencia lectora }\end{array}$ \\
\hline
\end{tabular}

Figura 2. Síntesis Programas de intervención

Fuente: De la Peña (2016b, p.312)

A continuación, se proponen ejemplos de programas de intervención que se pueden utilizar en el ámbito educativo para Dislexia:

Programa RAVE-O (Wolf, et al., 2000): trabaja velocidad de procesamiento y procesamiento fonológico, ortografía, memoria y conocer el significado de las palabras.

Programa Fast For Word: este programa a través de juegos entrena la fluidez, decodificación, conciencia fonológica, sintaxis, semántica y memoria de trabajo.

- Programa de estimulación sensorial integrada (Reynolds, Nicolson \& Hambly, 2003): este programa está basado en la teoría cerebelar - motora y está compuesto por ejercicios visomotores y vestibulares.

Programas de fluidez lectora (Khun \& Stahl, 2003; Serrano and Defior, 2012; Wexler, Vaughn, Roberts and Denton, 2010): estos programas trabajan la fluidez desde la lectura repetida y acelerada. fonológica.

Programa fonológico (Torgersen et al., 2001): entrena la conciencia

Programa con padres (Burns \& Kodrick, 1998): este programa implica a los padres en la terapia para los alumnos con dislexia mediante la reeducación de los errores disléxicos. 
La tecnología educativa proporciona una diversidad de programas que permiten trabajar de manera lúdica y divertida los distintos procesos afectados en Dislexia. Algunas de las herramientas tecnológicas dirigidas para escolares con Dislexia son las siguientes (De la Peña, 2014, 2015):

- AlphaWriter: aplicación que enseña el reconocimiento de consonantes y vocales, sus sonidos, el deletreo fonético y a escribir historias.

- Alphabet Zoo: aplicación que facilita el reconocimiento de los sonidos de las letras, utilizando texto e imágenes de animales.

- $\quad$ Graphogame: permite aprender las letras y sus sonidos.

- $\quad$ ACT Spell: trabaja el reconocimiento de palabras, la ortografía, la lectura y el control motor.

- Letter Reflex: aplicación para corregir las confusiones b,d,p,q,6,9 que presentan los disléxicos.

- Typ-0: aplicación que permite deletrear palabras ortográficamente.

- $\quad$ Sentence Builder: permite construir frases correctas gramaticalmente.

- Piruletras: conjunto de ejercicios basados en conciencia fonológica (omisión, sustitución e inversión de letras, derivación y separación de palabras).

- WoodLadder: juego de palabras que consiste en cambiar y formar nuevas palabras a partir de las dadas.

- Bob's Books: aplicación de juegos interactivos para trabajar la fonética, pronunciando palabras, deletreando y relacionando sonidos con palabras.

Tradislexia: videojuego en 3D diseñado para trabajar el procesamiento sintáctico, semántico y ortográfico, la conciencia fonológica y la percepción del habla a partir de 10 años de edad.

- Dyslexia Quest: juegos para entrenar la memoria visual y memoria verbal.

- Ikonmap: permite realizar mapas conceptuales facilitando el aprendizaje visual a los disléxicos.

- Dyslexia like me: libro electrónico que mediante un niño se aprende acerca de la dislexia y de nuevas herramientas para aprender. Trabaja desde un enfoque multisensorial (vista, sonido y tacto).

- Spelling Bee Challenge: consiste en un concurso simulado sobre ortografía que favorece el deletreo y el vocabulario.

En síntesis, la finalidad de la intervención neuropsicológica educativa en Dislexia es mejorar la situación académica y personal de este alumnado, ayudando a superar las dificultades que presentan, por lo que es imprescindible el trabajo conjunto de la familia, escolar disléxico, profesorado y profesionales externos.

\section{Referencias}

American Psychiatric Association (2013). DSM-5. Manual diagnóstico y estadístico de los trastornos mentales. Editorial Médica Panamericana.

Baldo, J. \& Dronkers, N. (2006). The role of inferior parietal and inferior frontal cortex in working memory. Neuropsychology, $20,529-538$.

Burns, G. \& Kodrick. P. (1998). Psychological behaviorism's Reading therapy program: parents as Reading therapists for their children's Reading disability. Journal of Learning Disabilities, 31(3), 278-285.

Cappa, S. (2015). Disorders of Language. Brain Mapping, 3, 1089-1093.

De la Peña, C. (2014). Herramientas tecnológicas para escolares con dislexia. Cuadernos de Pedagogía.

De la Peña, C. (2015). Programas para la Dislexia desde la base neuropsicológica. En P. Martín (Coord.), Procesos y programas de neuropsicología educativa (pp. 169-179). Madrid: Ministerio de Educación, Cultura y Deporte. 
De la Peña, C. \& Pradas, S. (2016). Dificultades del lenguaje y la detección de los procesos neuropsicológicos relacionados. En P. Martín y E. Vergara-Moragues, Procesos e instrumentos de evaluación neuropsicológica educativa (pp. 164-169). Madrid: Ministerio de Educación, Cultura y Deporte.

De Thorne, L., Hengst, J. \& Hamilton, M.-B. (2016). Communication Disorders. En H. Friedman (Ed.), Encyclopedia of Mental Health (Second Edition) (pp. 324-329). Academic Press.

Gallego, C. \& Rodríguez-Santos, F. (2009). Trastornos específicos del lenguaje. En AEPap (Ed.), Actualización Pediátrica 2009 (pp. 239-248). Madrid: Exlibris Ediciones.

Kuhn, M., Stahl, S. (2003). Fluency: A Review of Developmental and Remedial Practices. Journal of Educational Psychology, 95, 3-21. Recuperado de http://dx.doi.org/10.1037/0022-0663.95.1.3.

Meyler, A., Keller, T., Cherkassky, V., Gabrieli, J. \& Just, M. (2008). Modifying the brain activation of poor readers during sentence comprehension with extended remedial instruction: A longitudinal study of neuroplasticity. Neuropsychologia, 46, 2580-2592.

Moreno-Flagges, N. (2013). Trastornos del lenguaje. Diagnóstico y tratamiento. Rev Neurol, 57(S1), S85-S94.

Peña-Casanova, J. (2001). Manual de logopedia. Barcelona: Masson.

Pradas, S. \& De la Peña, C. (2015). Procesos y programas para superar las dificultades del lenguaje. En P. Martín (Coord.), Procesos y programas de neuropsicología educativa (pp. 187-199). Madrid: Ministerio de Educación, Cultura y Deporte.

Puyuelo, M. \& Salavera, C. (2011). Patología del lenguaje. Evaluación e intervención. Boletín Aelfa, 11(1), 33-37.

Reynolds, D., Nicolson, R. \& Hambly, H. (2003). Evaluation of an Exercise-based treatment for children with reading difficulties. Dyslexia, 9, 48-71.

Serrano F. \& Defior, S. (2012). Efficacy of RFI (Reading Fluency Intervention) program in Spanish dyslexic and poor readers across age. Comunicacion oral presentada en el Nineteenth Annual Meeting Society for the Scientific Study of Reading, Montreal, Canada 11-14 de Julio de 2012.

Shaywitz, B., Shaywitz S., Blachman B., Pugh K., Fulbright R., Skud- larski P., et al. (2004). Development of left occipitotemporal systems for skilled reading in children after a phonologically-based intervention. Biol Psychiatry, 55, 926-3.

Torgersen, J., Alexander, A., Wagner, R., Rashotte, C., Voeller, K., Conway, T. \& Rose, E. (2001). Intensive remedial instruction for children with severe reading disabilities: Inmediate and long-term outcomes from two instructional approaches. Journal of Learning Disabilities, $34,33-58$.

Wexler, J., Vaughn, S., Roberts, G. \& Denton, C. (2010). The efficacy of repeated reading and wide reading practice for high school students with severe reading disabilities. Learning Disabilities Research \& Practice 25, 2-10. Recuperado de http://dx.doi.org/10.1111/j.1540-5826.2009.00296.x

Wolf, M., Miller, L. \& Donnelly, K. (2000). Retrieval, Automaticity,Vocabulary, Elaboration, Ortografy (RAVE-O): Acomprehensive, fluency-based reading intervention program. Journal of Learning Disabilities, 33, 375-386. 